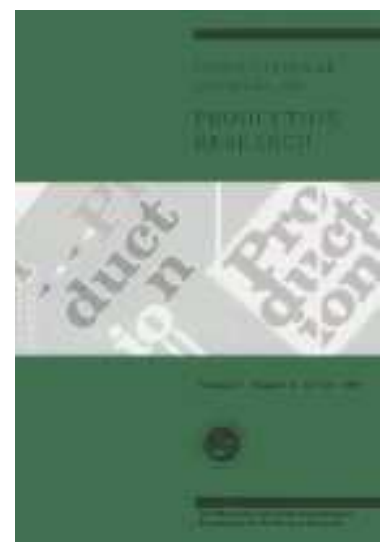

\title{
Dynamic Product Acquisition in Closed Loop Supply Chains
}

\begin{tabular}{|c|c|}
\hline Journal: & International Journal of Production Research \\
\hline Manuscript ID: & TPRS-2010-IJPR-0598.R1 \\
\hline Manuscript Type: & Original Manuscript \\
\hline $\begin{array}{r}\text { Date Submitted by the } \\
\text { Author: }\end{array}$ & $25-$ Oct-2010 \\
\hline Complete List of Authors: & $\begin{array}{l}\text { Minner, Stefan; University of Vienna, Department of Business } \\
\text { Administration } \\
\text { Kiesmuller, G; Eindhoven University of Technology, School of } \\
\text { Industrial Engineering }\end{array}$ \\
\hline Keywords: & REVERSE LOGISTICS, SUPPLY CHAIN MANAGEMENT \\
\hline Keywords (user): & Optimal control, Inventory \\
\hline
\end{tabular}

\section{SCHOLARONE}

Manuscripts 
International Journal of Production Research

Vol. 00, No. 00, 00 Month 2009, 1-22

\title{
Research Article
}

\section{Dynamic Product Acquisition in Closed Loop Supply Chains}

\author{
Stefan Minner ${ }^{a *}$ and Gudrun P. Kiesmüller ${ }^{b}$ \\ ${ }^{a}$ Faculty of Business, Economics and Statistics, University of Vienna, Austria; \\ ${ }^{b}$ Faculty of Economics and Social Sciences, Christian-Albrechts-Universität zu \\ Kiel, Germany
}

(October 2010)

\begin{abstract}
We consider a closed loop supply chain where demands can either be satisfied from manufacturing new products or by buying back used products from customers and upgrading their functionality by remanufacturing. A joint buy-back pricing and manufacturing-remanufacturing decision model at the operationsmarketing interface model is presented that allows for dynamic parameters, e.g., product life cycles and seasonal aspects. The model allows to identify beneficial opportunities for buying back and storing used products for immediate and future recovery. We present a new deterministic, dynamic, continuous time optimization model, derive necessary and sufficient optimality conditions, and develop a solution algorithm to find the cost minimizing manufacturing and remanufacturing policies as well as buy back strategies for used products based on Pontryagin's Maximum Principle. It is shown that an optimal policy, in general, will include time intervals where returns are acquired as to synchronize demand and remanufacturing, where returns are acquired and stored for future remanufacturing, and intervals where demand is satisfied by a mix of manufactured and remanufactured products. Furthermore, we discuss several reactive and proactive acquisition and remanufacturing heuristics and show under which conditions they are optimal. The findings are illustrated by numerical examples.
\end{abstract}

Keywords: Reverse logistics, Closed loop supply chain management, Inventory, Pricing, Optimal control

\footnotetext{
${ }^{*}$ Corresponding author. Email: stefan.minner@univie.ac.at
}

ISSN: $1478-6451$ print/ISSN 1478-646X online 


\section{Introduction}

In addition to manufacturing and distribution of new products, closed loop supply chains include the processes that take place after the use of a product at the end of its use. The incentives to move from forward to closed loop supply chains are legal ones, i.e. manufacturers are assigned responsible for their products after their usage, and economic ones, i.e. parts and materials of used products still have an economic value and can be used, e.g., as service parts after some remanufacturing and upgrading processes. As a consequence of the economic incentive, a second supply mode for parts and materials becomes available which increases the complexity of operations management. Strategic and operational aspects of return network design, redistribution channel choice, collection, quality and testing, disassembly, and reassembly have to be addressed and combined with forward processes.

Various quantitative models have been developed to provide decision support for these strategic and operational decisions, see Fleischmann et al. (1997), Fleischmann (2001), and Dekker et al. (2004) for overviews. The majority of the approaches faces two limitations. First, it is assumed that product returns/waste streams are passively accepted whereas there are practical needs for a product acquisition management (Guide and van Wassenhove 2001, Sasikumar and Kannan 2008) and there is empirical evidence that firms do acquisition management (Guide and Jayaraman 2000). Second, the majority of the models is static and therefore ignores life cycle aspects, seasonal aspects, trends, and in general time dependencies of model parameters (Minner and Kleber 2001). Instead of following a reactive strategy, to take back products at the end of their use, a proactive strategy appears to be promising. Instruments for such a strategy are to advertise return opportunities, i.e. create awareness for environmentally friendly products and firms, a buy back of used products as it is done, e.g., for toner cartridges, and to adjust contract design, e.g., for the lease of copiers.

A first model that addresses product acquisition management in a single period context is analyzed by Guide et al. (2003). The authors consider product returns that can be assigned to different quality classes. Within each class, customers are willing to return their product if a certain buy back price is offered. Therefore, returns for each quality class can be modeled by a return response function. The objective is to maximize the profit given by the sales of remanufactured products (determined from a sales price response function) minus the costs of acquiring respective returns and remanufacturing them. Guide et al. (2003) determine optimal acquisition and selling prices under the assumption of a perfect remanufacturing process. Bakal and Akcali (2006) drop this assumption and study the effects of random yield in the remanufacturing process. Galbreth and Blackburn (2006) allow for scrapping of products if the acquired products are too costly to remanufacture and derive the optimal acquisition and sorting policy. Pricing models for various degrees of reuse of recyclable components are presented in Vadde et al. (2007). They consider a single and multiple types of product returns and compare a proactive acquisition policy with a passive policy, where all returns are accepted. All these contributions have in common that they focus on a single period and therefore apply static models.

Dynamic models of pricing are used in the marketing literature to investigate diffusion of innovations (e.g., whether to use a skimming or penetration strategy) and to incorporate cost declines due to accumulated volume of production and learning effects (Simon 1989, Rao 1993). Kalish (1983) uses an optimal control approach in order to determine the optimal pricing policy for a monopolist, Dockner and Jørgensen (1988) analyze the 


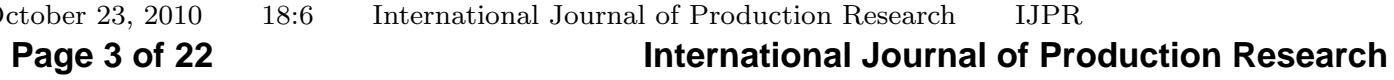

impact of advertising policies (see Feichtinger et al. (1994) for an overview) and Mesak and Clark (1998) investigate both marketing mix instruments simultaneously. A slightly different model with pricing and warranty period decisions is presented in Mesak (1996). For a general overview on pricing theory and practice we refer to Noble and Gruca (1999).

The main difference to the existing literature is that our model allows for dynamic demands and returns and recoverable items. Used products can be bought back under beneficial circumstances and stored for later use. As a consequence, the combined analysis of waste stream and product acquisition management is possible (Guide and van Wassenhove 2001). In contrast to other dynamic pricing models in marketing, we do not model spill over of marketing mix instruments, e.g. past pricing and customer expectations, effects of advertising, and competitive reactions. The only dynamic carry over that our model allows are inventories. This analysis is another contribution to the field of joint production and marketing models (see Eliashberg and Steinberg 1993).

In a dynamic model with product returns and remanufacturing, a different type of cost reductions appears. Returns of products and the opportunity to remanufacture them, which, in general, is assumed to be cheaper than manufacturing a new unit, offers a second supply mode (Minner 2003). However, this opportunity depends on the product's life cycle characteristics and the return behavior of the customers. In the following we analyze a dynamic model of product acquisition and recovery. In contrast to Guide et al. (2003) we assume that the demands cannot be influenced by pricing, i.e. the firm is a price taker on the sales side and that there is only a single market, i.e. we do not consider market segmentation effects between new and remanufactured products. Further, we consider a single quality level for returns which can be influenced by buy back offers according to a price/effort response function. Note that in the context of our model, buy back has a different meaning than in supply chain coordination where buy back (for new products not sold to customers) contracts are a mechanism to achieve channel coordination (see e.g. Lariviere 1999).

The paper is organized as follows. In Section 2 we present a static model for a single period problem and linear response functions. This illustration, which is a special case of the model analyzed in Guide et al. (2003), provides the basics of the effects that will appear in the dynamic model. Then, we present the additional assumptions for the dynamic model and give the optimal control optimization problem. In Section 3, we apply Pontryagin's Maximum Principle to state the necessary (and in our case also sufficient) conditions for an optimal solution. Using these conditions, we further characterize the optimal recovery and buy back policy. These results are exploited to develop an algorithm that constructs an optimal policy for arbitrary demand functions and parameters of a linear return response function. In Section 4 we discuss some simple heuristic policies and show some illustrative examples in Section 5. Finally, in Section 6, we summarize the main findings and discuss streams for further extensions of the framework.

\section{A static demand and acquisition model}

Consider a remanufacturer who is selling a single product over a single period. The customer demand $d>0$ for this product is deterministic. There are two options available for satisfying demand, manufacturing of $x$ units at a unit cost of $c_{p}$ and remanufacturing $u$ units at a unit cost of $c_{u}$. We assume that all demands have to be satisfied which leads to $d=x+u$. Due to legislation the remanufacturer is obliged to accept all offered returns. If more than the required $u$ units are returned, there is a disposal option where $z$ units 
are salvaged at a unit $\operatorname{cost} c_{z}$. This cost rate might be negative if there exist revenues from selling scrap. For example, the remanufacturer may sell the returns to a material recycling company.

Demands to be satisfied using the remanufacturing option require returns. The remanufacturer is applying a proactive acquisition policy and offers a price incentive to customers who return their used products. We assume that returns of used products can be initiated according to a return response function $r=f(p) \geq 0$, where $p \geq 0$ is the buy back price offered to customers that have the product in use. An alternative, more general, interpretation is that $p$ describes the level of effort put into product acquisition, i.e. advertising or paid transportation for returning the used item. Similar to Bakal and Akcali (2006) or Galbreth and Blackburn (2006), we assume a linear return response function

$$
f(p)=a+b p \quad a, b \geq 0
$$

which is the most simple type of response function found in marketing and economics literature. For other widely used and more general response functions we refer to Simon (1989). The number of returns consists of a number of autonomous, effort/price independent returns $a$ and a parameter $b$ that expresses the price sensitivity of customers. This implies that there are always some customers willing to return their products (if $a>0$ ) and that the return quantity increases with increasing acquisition effort.

The remanufacturer determines in the optimal buy back price as well as in the optimal quantities to remanufacture, produce or to dispose of in order to minimize cost. We can formulate the problem as a constrained non-linear minimization problem.

$$
\begin{aligned}
\min _{x, u, z, p} & c_{p} x+c_{u} u+c_{z} z+p(a+b p) \\
\text { s.t. } & x+u=d \\
& u+z=a+b p \\
& x, u, z, p \geq 0 .
\end{aligned}
$$

The first constraint (3) ensures that demand is satisfied while the second constraint (4) reflects that all returns are either remanufactured or disposed of. Obviously, all decision variables have to be non-negative. We can reduce the problem size using $x=d-u$ and $z=a+b p-u$. Then, the equivalent optimization problem with price and remanufacturing quantity as decision variables is obtained.

$$
\begin{aligned}
\min _{p, u} & c_{p}(d-u)+\left(c_{u}+p\right) u+\left(c_{z}+p\right)(a+b p-u) \\
\text { s.t. } & u \leq d \\
& u \leq a+b p \\
& p, u \geq 0 .
\end{aligned}
$$

The optimal solution of this problem is characterized in Theorem 2.1:

Theorem 2.1: The optimal buy back and recovery strategy depends on the recovery cost advantage $c_{p}-c_{u}$ and the disposal cost $c_{z}$. The optimal decisions $p^{*}, x^{*}, u^{*}, z^{*}$ are

- Region $A$ : $-c_{z} \leq(2 d-a) / b, c_{p}-c_{u} \geq(2 d-a) / b, a<d$ 
$p^{*}=(d-a) / b, x^{*}=0, u^{*}=d, z^{*}=0$

- Region B: $(2 d-a) / b>c_{p}-c_{u}>a / b, c_{p}+c_{z}-c_{u} \geq 0,-c_{z} \leq(2 d-a) / b, a<d$ $p^{*}=\frac{b\left(c_{p}-c_{u}\right)-a}{2 b}, x^{*}=d-u^{*}, u^{*}=\frac{a+b\left(c_{p}-c_{u}\right)}{2}, z^{*}=0$

- Region C: $c_{p}+c_{z}-c_{u} \geq 0, c_{p}-c_{u} \leq \frac{a}{b}, a<d$ $p^{*}=0, x^{*}=d-a, u^{*}=a, z^{*}=0$

- Region D: $c_{p}+c_{z}-c_{u} \geq 0, c_{p}-c_{u} \geq(2 d-a) / b,-c_{z} \geq(2 d-a) / b$ $p^{*}=-\left(a+b c_{z}\right) /(2 b), x^{*}=0, u^{*}=d, z^{*}=a+b p-d$

- Region $E: c_{p}+c_{z}-c_{u} \leq 0,-c_{z} \leq a / b$ $p^{*}=0, x^{*}=d, u^{*}=0, z^{*}=a$

- Region F: $c_{p}+c_{z}-c_{u} \leq 0,-c_{z}>a / b$ $p^{*}=-\left(a+b c_{z}\right) /(2 b), x^{*}=d, u^{*}=0, z^{*}=\frac{a-b c_{z}}{2}$

- Region $G: a \geq d, c_{p}-c_{u} \geq-c_{z},-c_{z} \leq \frac{a}{b}$ $p^{*}=0, x^{*}=0, u^{*}=d, z^{*}=a-d$

The proof of Theorem 2.1 is presented in Appendix A. For the case of practical interest where $d>a$, Figure 1 illustrates these regions.

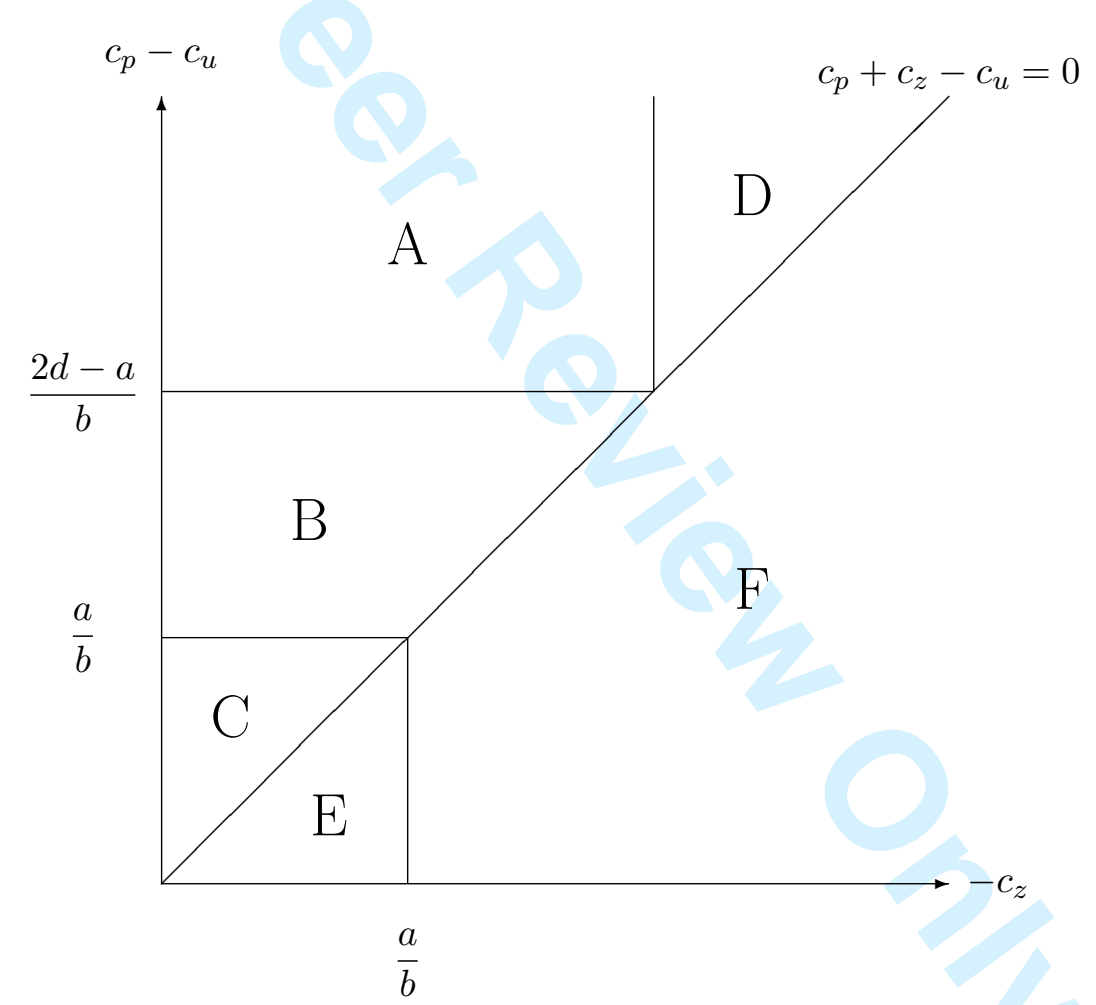

Figure 1. Illustration of regions for $d>a$

In cases where remanufacturing (without considering acquisition) is generally profitable, i.e. where the cost of disposing a return and manufacturing a new item is more expensive than remanufacturing $\left(c_{p}+c_{z}>c_{u}\right)$, we have different policies being separated by threshold values for the recovery cost advantage $c_{p}-c_{u}$. If the recovery cost advantage is positive but low $c_{p}-c_{u} \leq \frac{a}{b}$, it is optimal to remanufacture all autonomous returns $a$, not to buy additional returns $p=0$ and to manufacture the remaining demand $d-a$ (Case C). If the recovery cost advantage is sufficiently large $c_{p}-c_{u} \geq \frac{2 d-a}{b}$, it becomes 


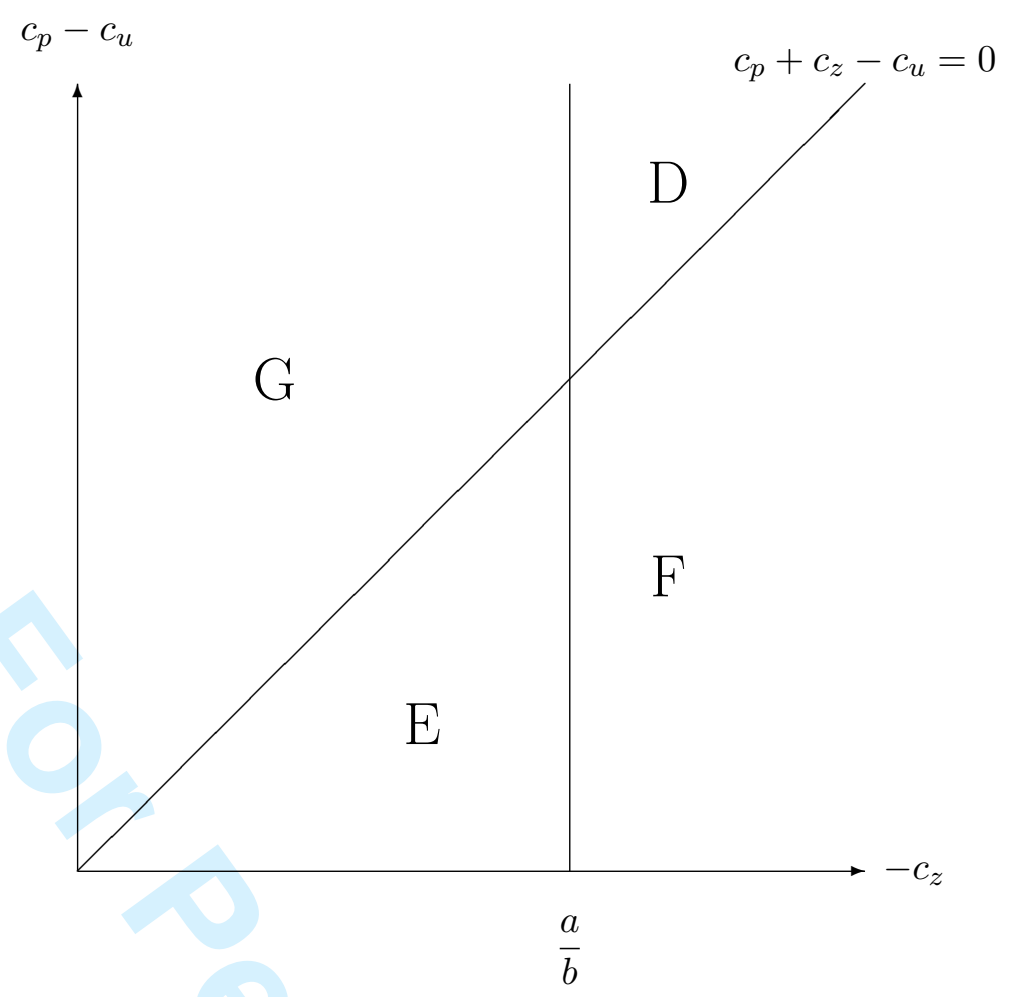

Figure 2. Illustration of regions for $d \leq a$

optimal to buy back as many returns as being required to satisfy demand from recovery products in full (Region A). If further the salvage value is positive and large enough $\left(-c_{z} \geq \frac{2 d-a}{b}\right)$, further returns are acquired and beneficially salvaged (Region D). For intermediate recovery cost advantages $\frac{a}{b}<c_{p}-c_{u}<\frac{2 d-a}{b}$, it is optimal to buy returns only at a maximum price, to remanufacture all returns and to manufacture the remaining demand.

The threshold values are defined as follows: $c_{p}-c_{u}=\frac{a}{b}$ defines the recovery advantage where the optimal price becomes zero and $c_{p}-c_{u}=\frac{2 d-a}{b}$ determines the buy back price such that returns equal demands. If autonomous returns $a$ are larger or equal to the demand (see Figure 2), we find that regions $\mathrm{E}$ and $\mathrm{F}$ remain. If $c_{p}+c_{z}-c_{u} \geq 0$ but salvaging returns is not beneficial enough $\left(-c_{z} \leq \frac{a}{b}\right)$, it is optimal to remanufacture for demand and to dispose the remaining autonomous returns (Region $G$ ) whereas if $-c_{z}>\frac{a}{b}$ remanufacturing for demand and salvaging of excessive returns (Region $\mathrm{D}$ ) is optimal.

\section{A dynamic demand and acquisition model}

Seasonal and life cycle demand is common in Supply Chains. In order to represent these dynamics over a finite planning horizon $t \in[0, T]$, we model demand during the selling season as a continuous and differentiable function of time $(d(t) \geq 0, t \in[0, T])$. In order to match supply with demand, the remanufacturing facility needs different quantities of returns at different moments in time. Acquiring the right amount of returns is aggravated by dynamic supply. However, during periods where there is not enough supply, the remanufacturer can encourage customers to return their products by setting economic 
incentives. We assume a dynamic buy back price function $p(t)$, and continuous and differentiable functions $a(t), b(t)$, resulting in a time dependent return response function $r(t)=a(t)+b(t) p(t)$.

If supply of returns exceeds demand, the remanufacturer has, besides the disposal option, the possibility to keep returned products in recoverables inventory which is subject to out of pocket inventory holding cost rate $h$ for recoverables $y(t) \geq 0$. Opportunity costs for capital investment are incorporated by discounting payments with a constant discount rate of $\rho$.

In order to avoid trivial solutions, we make the following assumptions concerning the cost structure.

$(\alpha 1)$ Remanufacturing a unit is always cheaper than disposing of a return and manufacturing a new one $\left(c_{p}+c_{z}-c_{u}>0\right)$. Otherwise the optimization can be decoupled in a way that all demands are manufactured and all returns are disposed of (see Regions $\mathrm{E}$ and $\mathrm{F}$ in the static analysis).

$(\alpha 2)$ It is not advantageous to buy returns for disposal $\left(-c_{z} \leq a(t) / b(t)\right)$.

$(\alpha 3)$ The interest benefit from delaying disposal is smaller than the holding cost $\left(\rho c_{z}<\right.$ $h$ ). Otherwise unnecessary units would never be disposed of but kept in inventory.

The state of the system to be controlled is recoverable inventory $y(t)$. Initial and final inventory levels are assumed to be zero, $y(0)=y(T)=0$. The manager has to decide when to build up recoverables inventory and when to stop economic incentives for product returns. Additionally, he has to determine the amounts to be remanufactured and to be disposed of. The associated movement of the state in continuous time is

$$
\dot{y}(t)=r(t)-u(t)-z(t) \quad \forall t \in[0, T]
$$

where $\dot{y}(t)$ denotes the first derivative of the function $y(t)$ with respect to time. The objective is to minimize total discounted $\operatorname{cost} C$ over a finite planning horizon of length $T$ subject to satisfying all given demands.

$$
C=\int_{0}^{T} e^{-\rho t}\left(c_{p} x(t)+c_{u} u(t)+c_{z} z(t)+p(t) r(t)+h y(t)\right) d t
$$

Under the assumption that demand always has to be satisfied and cannot be backordered we can replace $x(t)=d(t)-u(t)$ and the remaining control variables at each instant of time are the price $p(t)$ for returns, the remanufacturing decision $u(t)$, and the disposal decision $z(t)$. The resulting optimal control problem is

$$
\begin{aligned}
& \min _{p, u, z} \int_{0}^{T} e^{-\rho t}\left(\left(c_{u}-c_{p}\right) u(t)+c_{z} z(t)+p(t)(a(t)+b(t) p(t))+h y(t)\right) d t \\
& \text { s.t. } \\
& 0 \leq u(t) \leq d(t) \quad t \in[0, T] \\
& \dot{y}(t)=a(t)+b(t) p(t)-u(t)-z(t) \quad t \in[0, T] \\
& y(0)=y(T)=0 \\
& y(t) \geq 0, p(t) \geq 0, u(t) \geq 0, z(t) \geq 0 \quad t \in[0, T] .
\end{aligned}
$$


Table 1. Optimal pricing and recovery policy for state $y(t)=0$

\begin{tabular}{cccc}
\hline Condition & $d(t)<\frac{a(t)}{2}-c_{z} \frac{b(t)}{2}$ & $\begin{array}{c}a(t) \\
2\end{array}$ & $\begin{array}{c}c_{z} b(t) \\
\text { and }\end{array}$ \\
& $d(t) \leq \frac{a(t)}{2}+\frac{b(t)\left(c_{p}-c_{u}\right)}{2}$ & $d(t)>\frac{a(t)}{2}+\frac{b(t)}{2}\left(c_{p}-c_{u}\right)$ \\
\hline Solution & $u^{*}(t)=d(t)$ & $u^{*}(t)=d(t)=r^{*}(t)$ & $u^{*}(t)=r^{*}(t)$ \\
& $x^{*}(t)=0$ & $x^{*}(t)=0$ & $x^{*}(t)=d(t)-u(t)$ \\
& $z^{*}(t)=r(t)-d(t)$ & $z^{*}(t)=0$ & $z^{*}(t)=0$ \\
& $p^{*}(t)=0$ & $p^{*}(t)=\frac{d(t)-a(t)}{b(t)}$ & $p^{*}(t)=\max \left\{0, \frac{b(t)\left(c_{p}-c_{u}\right)-a(t)}{2 b(t)}\right\}$ \\
$\lambda^{*}(t)=-c_{z}$ & $\lambda^{*}(t)=\frac{2 d(t)-a(t)}{b(t)}$ & $\lambda^{*}(t)=c_{p}-c_{u}$ \\
\hline
\end{tabular}

\subsection{Optimal acquisition and remanufacturing policy}

In order to derive the optimal decisions we split the analysis into three parts. We first present the situation where no returns are kept in inventory. Then we analyze the optimal manufacturing and pricing decisions under positive inventories, and in the last step we combine both situations and their transitions.

Theorem 3.1: Given a state where the inventory level is equal to zero $y(t)=0$ and not leaving this state, the optimal decisions are given in Table 1.

Proof: See Appendix B.

As in the static case, the optimal decisions depend on the disposal costs $c_{z}$ and the cost difference between manufacturing and remanufacturing $\left(c_{p}-c_{u}\right)$. Since inventory is empty and it is never beneficial to buy returns for disposal $(\alpha 1)$, it is only optimal to dispose of items when autonomous returns are larger than demand and the company is already getting more used products than needed (first column of Table 1). This case corresponds to Region $\mathrm{D}$ and $a>d$ in the static case. If the difference between manufacturing and remanufacturing costs is relatively large, then complete synchronization of supply and demand, as described in column 2 of Table 1, is optimal. This case corresponds to region A in the static model. Manufacturing can be optimal in two different situations. First, if the remanufacturing advantage is small $\left(c_{p}-c_{u} \leq a(t) / b(t)\right)$, then it is optimal to set the price equal to zero and just to remanufacture autonomous returns (Region $\mathrm{C}$ in the static model). On the other hand manufacturing may be required if not enough returns can be bought at a maximum economical price. (Region B in the static model). Taking a different perspective from the magnitude of demand, small demands $d(t)$ are satisfied from autonomous demands only (if possible), for intermediate demands, return and demand can be synchronized by setting an appropriate buy-back price, whereas for large demands, returns are remanufactured up to an economical level and the remaining demands are manufactured.

Furthermore, $\lambda^{*}(t)$ has an economic interpretation as the (marginal) value of an additional return at $t$. If returns are disposed, the value equals the salvage value/disposal cost $\lambda=-c_{z}$. In the synchronization case, $\lambda=\frac{2 d(t)-a(t)}{b(t)}$, i.e., the value follows the development of the demand and buy-back conditions whereas in case manufacturing is required, $\lambda(t)=c_{p}-c_{u}$ and the value equals the recovery cost advantage.

Under the assumption of positive recoverables inventory and no transition to the zero inventory state, only remanufacturing for demand is optimal.

Theorem 3.2: Given the inventory level is positive $(y(t)>0)$ and not leaving this 
state, the optimal decisions are:

$$
\begin{aligned}
& u^{*}(t)=d(t), x^{*}(t)=0, z^{*}(t)=0 \\
& p^{*}(t)=\max \left\{0, \frac{\lambda(t)}{2}-\frac{a(t)}{2 b(t)}\right\} \text { with } \lambda(t)=-\frac{h}{\rho}+\left(\lambda\left(t_{0}\right)+\frac{h}{\rho}\right) e^{\rho\left(t-t_{0}\right)}<c_{p}-c_{u}
\end{aligned}
$$

Proof: See Appendix C.

Intuitively, disposing of products simultaneously with building up inventories cannot be optimal. Similarly, there is no need for more expensive manufacturing as long as there is inventory of returns. Note that the optimal buy back price can be expressed as a function of the adjoint variable $\lambda(t)$ which is the shadow price (value) of an additional return at time $t$. Note that time $t_{0}$ denotes either the start or end of an interval with positive inventory. Since there is a maximal buy-back price which is economically reasonable, we have an upper bound for the number of returns to be acquired $r^{*}(t)<\frac{a(t)+b(t)\left(c_{p}-c_{u}\right)}{2}$.

Besides the optimal decisions in one state we require the optimal moments in time of transitions, especially when to start collecting returns for building up recoverables inventory. Let $t_{s}$ denote the start of an optimal time interval with positive inventory and $t_{e}$ the end of such an interval.

Proposition 3.3: $\quad$ (Collection conditions) For optimal time intervals $\left[t_{s}, t_{e}\right]$ with positive inventories $(y(t)>0)$ the following conditions have to hold:

$$
\begin{array}{r}
\int_{t_{s}}^{t_{e}}\left(d(s)-\left(a(s)+b(s) p^{*}(s)\right) d s=0\right. \\
\int_{t_{s}}^{t}\left(d(s)-\left(a(s)+b(s) p^{*}(s)\right) d s<0 \quad t_{s}<t<t_{e}\right.
\end{array}
$$

Over the entire interval, cumulative returns have to equal cumulative demands (such that inventory depletes to zero at the end of the interval). For any time $t$ within the interval, inventory has to be positive, i.e. cumulative returns up to $t$ have to exceed cumulative demands up to $t$.

There are two main reasons for collecting returns to build up recoverables inventory. First, (see Proposition 3.4) when customers are highly motivated to return used products and there is excess of autonomous returns $(a(t)>d(t))$, the remanufacturer has to chose between the two options of disposing of returned items or keeping products in stock for later use. The optimal decision depends on the disposal cost parameter $c_{z}$ and the return response function.

Proposition 3.4: (Excess autonomous returns) Let us consider time points $\theta$ with $d(\theta)=a(\theta), d\left(\theta-\epsilon_{1}\right)<a\left(\theta-\epsilon_{1}\right)$, and $d\left(\theta+\epsilon_{2}\right)>a\left(\theta+\epsilon_{2}\right)$ for small values of $\epsilon_{1}, \epsilon_{2}>0$. The optimal inventory at time $\theta$ has to satisfy the following condition:

$$
y^{*}(\theta)\left\{\begin{array}{c}
>0 \quad \text { if } \quad \frac{2 d(t)-a(t)}{b(t)}>-c_{z} ; \quad t \in\left(\theta-\epsilon_{1}, \theta\right) \\
=0 \quad \text { if } \quad \frac{2 d(t)-a(t)}{b(t)} \leq-c_{z} ; \quad t \in\left(\theta-\epsilon_{1}, \theta\right)
\end{array} .\right.
$$

Proof: See Appendix D.

Note that the two cases of (11) correspond to the separation of Regions A and D in 
the static model. If salvaging of returns is beneficial, no inventories are built whereas if synchronization of returns with demands has a larger value than disposal, than excessive items are collected.

The second reason for building up inventories (see Proposition 3.5) is motivated by a required price increase in order to acquire the demand quantity related to the costs for buying in advance and storing the item. Instead of synchronizing demand and returns the remanufacturing facility can acquire more returns and build up recoverable inventory.

Proposition 3.5: $\quad$ (Economic incentives) Let us assume that demand satisfies the following inequality

$$
\frac{a(t)}{2}-c_{z} \frac{b(t)}{2} \leq d(t)<\frac{a(t)}{2}+\frac{b(t)}{2}\left(c_{p}-c_{u}\right)
$$

For all time points $\theta$ satisfying

$$
\frac{b(\theta)(2 \dot{d}(\theta)-\dot{a}(\theta))-2 d(\theta) \dot{b}(\theta)-a(\theta) \dot{b}(\theta)}{b^{2}(\theta)}>\rho \frac{d(\theta)-a(\theta)}{b(\theta)}+h
$$

and (12), the optimal inventory has to be positive $y^{*}(\theta)>0$.

Proof: See Appendix E.

This situation occurs if demands and returns are synchronized, Region B in the static model, second column in Table 1). If due to the dynamic of the parameters $a(t), b(t)$, or $d(t)$ further synchronization is more expensive (expressed through the left-hand side in (13) which is the slope of $\frac{2 d(t)-a(t)}{b(t)}$, than buying in advance and storing, expressed through the right-hand side, becomes beneficial and results in positive inventory.

\subsection{Algorithm}

For the computation of the optimal price and recovery policy we have to determine the optimal time intervals of zero and positive inventory, respectively the starting points for building up inventories. In the following we sketch an algorithm for constructing the optimal solution. We start with an initial solution as given in Table 1 which means, we assume the three subcases for $y(t)=0$ are the status quo. Transitions from having zero recoverables inventory into collecting returns is required in order to satisfy the necessary optimality conditions as stated in Proposition 3.4 and 3.5.

The construction of optimal time intervals $\left[t_{s}^{*}, t_{e}^{*}\right]$ with positive recoverable inventory due to excess autonomous returns starts with determining intervals $\left[t_{s}^{(0)}, t_{e}^{(0)}\right]$ with $d(t)<$ $a(t) \forall t \in\left(t_{s}^{(0)}, t_{e}^{(0)}\right), d\left(t_{s}^{(0)}\right)=a\left(t_{s}^{(0)}\right)$ and $d\left(t_{e}^{(0)}\right)=a\left(t_{e}^{(0)}\right)$. Since the shadow price $\lambda(t)$ has to be continuous at the end of the interval, and based on Proposition 3.3 and 3.4, the following procedure computes the optimal interval $\left[t_{s}^{*}, t_{e}^{*}\right]$ :

STEP 1: Choose $t_{e}>t_{e}^{(0)}$ and compute $\lambda\left(t_{e}\right)$ as

$$
\lambda\left(t_{e}\right)=\min \left\{c_{p}-c_{u}, \frac{2 d\left(t_{e}\right)-a\left(t_{e}\right)}{b\left(t_{e}\right)}\right\}
$$

STEP 2: Find a $t_{s}<t_{e}$ such that the integral conditions (9) and (10) are satisfied. 
STEP 3: Compute $\lambda\left(t_{s}\right)$ as

$$
\lambda\left(t_{s}\right)=-\frac{h}{\rho}+\left(\lambda\left(t_{e}\right)+\frac{h}{\rho}\right) e^{\rho\left(t_{s}-t_{e}\right)}
$$

STEP 4: If $t_{s} \leq t_{s}^{(0)}$ and $\lambda\left(t_{s}\right)=\frac{2 d\left(t_{s}\right)-a\left(t_{s}\right)}{b\left(t_{s}\right)}$, then STOP.

If $t_{s}>t_{s}^{(0)}$ and $\lambda\left(t_{s}\right)=-c_{z}$, then STOP.

If $t_{s}>t_{s}^{(0)}$ and $\lambda\left(t_{s}\right)>-c_{z}$, we have to increase $t_{e}$. If $\lambda\left(t_{s}\right)<-c_{z}$, a smaller value of $t_{e}$ is required and the procedure continues with STEP 1 and the new value for $t_{e}$.

Similarly, we find the starting points of building up inventories due to economic incentives using Proposition 3.5 and applying an iterative procedure. We first determine intervals $\left[t_{1}, t_{2}\right]$ with

$$
\frac{b\left(t_{i}\right)\left(2 \dot{d}\left(t_{i}\right)-\dot{a}\left(t_{i}\right)\right)-2 d\left(t_{i}\right) \dot{b}\left(t_{i}\right)-a\left(t_{i}\right) \dot{b}\left(t_{i}\right)}{b^{2}\left(t_{i}\right)}=\rho \frac{d\left(t_{i}\right)-a\left(t_{i}\right)}{b\left(t_{i}\right)}+h \quad i=1,2
$$

and (13) holds for all $\theta \in\left(t_{1}, t_{2}\right)$. Next we have to calculate $\left[t_{3}, t_{4}\right]$ with

$$
d\left(t_{3}\right)=\frac{a\left(t_{3}\right)}{2}-c_{z} \frac{b\left(t_{3}\right)}{2} \text { and } d\left(t_{4}\right)=\frac{a\left(t_{4}\right)}{2}+\frac{b\left(t_{4}\right)}{2}\left(c_{p}-c_{u}\right)
$$

The following algorithm starts with the initial interval $\left[t_{s}^{(0)}, t_{e}^{(0)}\right]=\left[t_{1}, t_{2}\right] \cap\left[t_{3}, t_{4}\right]$ and constructs intervals with positive recoverable inventories based on Proposition 3.3 and utilizing the continuity of the shadow price $\lambda(t)$ at the interval boundaries.

STEP 1: Choose $t_{e}>t_{e}^{(0)}$ and compute $\lambda\left(t_{e}\right)$ from (14).

STEP 2: Compute $t_{s}<t_{e}$ using (15) and

$$
\lambda\left(t_{s}\right)=\frac{2 d\left(t_{s}\right)-a\left(t_{s}\right)}{b\left(t_{s}\right)} .
$$

STEP 3: Evaluate the integral as given in (9).

STEP 4: If the value of the integral is equal to zero, then STOP. If it is positive, then there is an excess of demands and $t_{e}$ has to be decreased. Otherwise, if the value of the integral is negative, there are too many returns and we have to increase $t_{e}$. Continue the procedure with STEP 1 and the new value for $t_{e}$.

These procedures can easily be implemented because only integrals have to be computed and equations have to be solved within a search and bisection scheme.

\section{Heuristic strategies}

Instead of deriving and implementing the optimal policy, alternative heuristic strategies can be applied. The first two heuristics are using a reactive acquisition policy, while heuristic three and four are based on a proactive acquisition policy. We derive conditions, depending on the cost parameters $c_{p}, c_{u}$, and $c_{z}$, and the return response function under which these simple strategies provide us with the optimal solution. The propositions in the following can easily be derived using Theorems 3.1 and 3.2 . 


\section{No buy back of returns, zero inventory (AUTO1)}

Under this strategy, we only collect and remanufacture a maximum of $a(t)$ units. The buy back price is always equal to zero $(p(t)=0)$. If $d(t)<a(t)$, excess returns are immediately disposed of, i.e. $z(t)=\max \{0, a(t)-d(t)\}$, otherwise manufacturing is used to obtain missing items to satisfy demand, i.e. $x(t)=\max \{0, d(t)-a(t)\}$, and $u(t)=\min \{d(t), a(t)\}$.

Proposition 4.1: If $d(t)>a(t)$ and $c_{p}-c_{u} \leq a(t) / b(t)$ for all $t \in[0, T]$, then heuristic AUTO1 is optimal.

\section{No buy back of returns, collect excess autonomous returns (AUTO2)}

All returns are collected and no additional price incentives are offered by the remanufacturer to increase the return quantities. Thus, the buy back price is always equal to zero $p(t)=0$. Excess returns $(a(t)>d(t))$ are kept in inventory and disposal does not take place $(z(t)=0)$. Manufacturing and remanufacturing quantities depend on whether there is recoverables inventory available or not.

$$
\begin{aligned}
& y(t)=0: x(t)=\max \{0, d(t)-a(t)\}, u(t)=\min \{d(t), a(t)\} \\
& y(t)>0: x(t)=0, u(t)=d(t)
\end{aligned}
$$

If there is positive recoverables inventory, no items are manufactured and demands are met from remanufactured units only. In case of zero inventory, the minimum of demand and returns is remanufactured and missing items are manufactured.

Proposition 4.2: If $c_{p}-c_{u} \leq a(t) / b(t)$ for all $t \in[0, T]$ and no excessive return has to be stored longer than beneficial, then heuristic AUTO 2 is optimal.

\section{Synchronization of returns and demands (SYN)}

Consider a strategy aiming at a complete synchronization of supply of returns and demand. The buy back price is therefore dynamically adapted such that as many returns as needed are acquired $p(t)=\max \{0,(d(t)-a(t)) / b(t)\}$. Demand is completely satisfied from remanufactured items $x(t)=0, u(t)=d(t)$. Additionally, no returned units are kept on stock $(y(t)=0)$, and excessive returns are immediately disposed of $z(t)=$ $\max \{0, a(t)-d(t)\}$.

Proposition 4.3: If $a(t) \leq d(t)$ for all $t \in[0, T]$ and the following two conditions hold for all $t \in[0, T]$

$$
\begin{array}{r}
-c_{z} \leq(2 d(t)-a(t)) / b(t) \leq c_{p}-c_{u} \\
\text { and } \frac{b(t)(2 \dot{d}(t)-\dot{a}(t))-2 d(t) \dot{b}(t)-a(t) \dot{b}(t)}{b^{2}(t)} \leq \rho \frac{d(t)-a(t)}{b(t)}+h
\end{array}
$$

then heuristic SYN is optimal.

\section{Optimal static policy (STA)}

Another simple approach is to implement the optimal static decision for each instant of time. Therefore, no inventories are required and the policy is given in Section 2.

Proposition 4.4: If i) (19) and (20) hold or if ii) $(2 d(t)-a(t)) / b(t) \geq c_{p}-$ $c_{u}$ for all $t \in[0, T]$, then heuristic STA is optimal. 


\section{Numerical examples}

We illustrate our results using the following seasonal demand pattern:

$$
d(t)=20+10 \sin (t-\pi), t \in[0,4 \pi]
$$

Demand varies between 10 and 30 units, i.e. $10 \leq d(t) \leq 30$. The discount rate is $\rho=0.01$, i.e. $1 \%$ continuous interest per unit of time which in our case is, e.g., a month. For the out of pocket holding cost rate we have chosen $h=0.05$.

Example 1: $a=0, b=5, c_{p}=10, c_{u}=4$ and $c_{z}=0$

Since there are no autonomous returns we only have to determine starting points for building up inventories when buying returns in advance and storing them is less expensive than manufacturing or increasing the buy-back price.

According to Theorem 2 we have to solve the following equation in order to compute the intervals where manufacturing is required in case of no stockkeeping.

$$
20+10 \sin (t-\pi)=15
$$

the resulting intervals are $[0,0.5236],[2.6180,6.8068],[8.9012,12.5664]$. In order to identify time points satisfying (13) we have to solve

$$
4 \cos (t-\pi)=0.01 \frac{2(20+10 \sin (t-\pi))}{5}+0.05
$$

resulting in $[1.5933,4.6699],[7.8765,10.9531]$. This leads to the following intervals where synchronization of returns and demands does not satisfy the necessary optimality conditions: $[1.5933,2.6180]$ and $[7.8765,8.9012]$. At least during these intervals recoverables inventory has to be positive. These initial intervals are enlarged according to the algorithm described above such that the adjoint variable is a continuous function and the collection conditions of Proposition 4 hold. In the optimal solution, inventory is held during $[0.6226,3.6935]$ and $[6.9058,9.9760]$. Therefore, the optimal pricing and recovery policy is as follows:

i) During the time interval $[0,0.5236]$ buy used items at a constant rate $r=15$ for $p_{\max }=3$, remanufacture them and produce for the remaining demands.

ii) During the time interval $[0.5236,0.6226]$ buy as many returns as being required to meet demand from remanufactured units.

iii) During the time interval $[0.6226,3.6935]$ build up a recoverable inventory and use returns and, if necessary, items from the recoverable inventory to satisfy demands.

iv) During the time interval $[3.6935,2 \pi]$ buy returns at a constant rate $r=15$ for $p_{\max }=3$ and manufacture for remaining demands.

v) In the interval $[2 \pi, 4 \pi]$ the optimal policy is the same as in $[0,2 \pi]$.

For a graphical illustration of the optimal recovery policy, see Figure 3 and for the optimal price, Figure 4.

For the comparison of the optimal strategy with the heuristics mentioned in Section 4 we introduce the relevant costs $C^{r e l}$, since we cannot avoid satisfying demand at least from remanufactured units. Subtracting the portion of the costs which cannot be influenced leads to the following definition of the relevant costs $C^{r e l}=C-c_{u} \int_{0}^{T} d(s) d s$. 


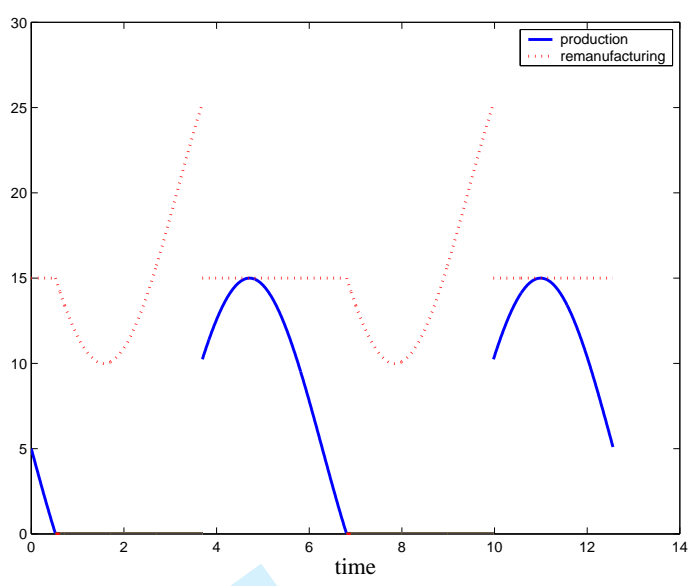

Figure 3. Optimal recovery policy

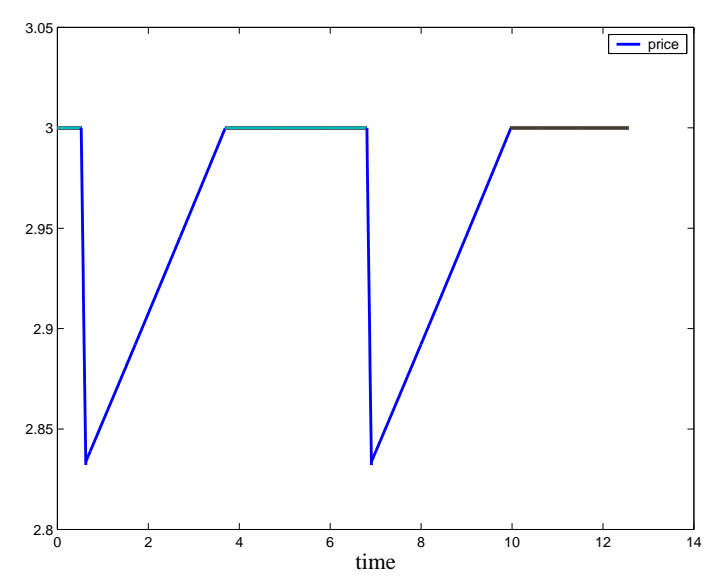

Figure 4. Optimal price policy

Table 2. Relevant costs heuristics

\begin{tabular}{lccccc}
\hline & Optimal & AUTO1 & AUTO2 & SYN & STA \\
\hline $\mathrm{b}=2.5$ & 1180.22 & & & 2172.86 & 1180.22 \\
\hline $\mathrm{b}=5$ & 908.17 & 1454.26 & 1454.26 & 1086.43 & 916.87 \\
\hline $\mathrm{b}=10$ & 487.93 & & & 543.21 & 543.21 \\
\hline
\end{tabular}

In order to illustrate the impact of different return response function parameters, we investigate different settings for $b$. The relevant costs are given in Table 2 .

For $b=2.5$ the static policy is optimal since $c_{p}-c_{u}<(2 d(t)-a(t)) / b(t)$ for all $t$. Returns are bought back with a constant rate of $r=7.5$ units at a price of $p_{\max }=3$ and the remaining demands are satisfied by production. In case of $b=10$, it is never optimal to produce. The synchronization infeasibility interval is $[1.606,4.657]$, the interval with positive inventory becomes $[0.133,6.145]$.

These examples show the importance of the reaction parameter $b$ on the minimal cost. A higher return response to increasing prices or acquisition efforts significantly reduces costs. Therefore, actions that influence this parameter, e.g. increasing visibility of buy back offers via Internet/E-commerce, creating customer awareness of buy back activities, paid return shipments will be promising.

Example 2: $a=15, b=5, c_{p}=10, c_{u}=8, c_{z}=1, h=1, \rho=0.01$

This example illustrates the case where intervals with excess autonomous returns become relevant. For this specific setting of parameters we find that $c_{p}-c_{u} \leq a / b$ and therefore $p(t)=0$ for all $t \in[0, T]$, i.e. it is never optimal to engage in active acquisition efforts. We obtain the following intervals where positive inventory is required due to economic incentives (Proposition 3.5):

$$
[1.8264,2.2935] \text { and }[8.1096,8.5767]
$$

Time intervals with less demand than autonomous returns are given by [0.5236,2.6180] and $[6.8068,8.9012]$. Since these intervals include the intervals given in $(24)$, we start with the construction of intervals with positive inventory due to excess autonomous returns. Since the cost for storing returns from $t=0.5236$ offset the savings of $c_{p}+c_{z}$ over $c_{u}$ 
the optimal solution starts keeping returns at $t_{s}=0.8029$. In the optimal solution the inventory is running empty at $t_{e}=3.7883$ and therefore, the inventory is positive also for the intervals given in (24). The optimal policy is the following:

i) Remanufacture autonomous returns with rate $a(t)$ and manufacture the remaining demand in $[0,0.5236]$.

ii) Remanufacture for demand and dispose excess returns in [0.5236, 0.8029].

iii) Remanufacture for demand and collect excessive returns respectively take required recoverables from inventory in [0.8029, 3.7883].

iv) Remanufacture autonomous returns and manufacture remaining demand $[3.7883,2 \pi]$.

v) In the interval $[2 \pi, 4 \pi]$ the optimal policy is the same as in $[0,2 \pi]$.

In Figure 5, the optimal manufacturing, remanufacturing, and disposal policy is depicted. As a consequence of switching from manufacturing to disposal at $t=0.5236$ and $t=6.8068$ the adjoint variable has a downward jump from $c_{p}-c_{u}$ to $-c_{z}$, for an illustration see Figure 6.

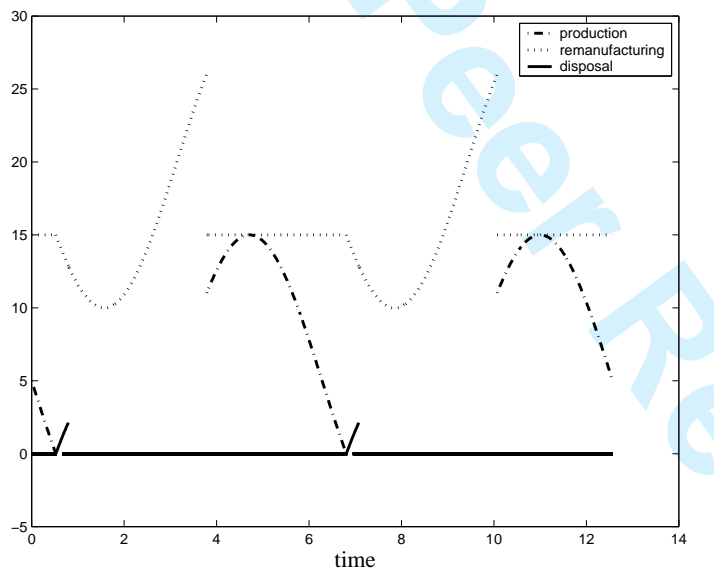

Figure 5. Optimal recovery policy

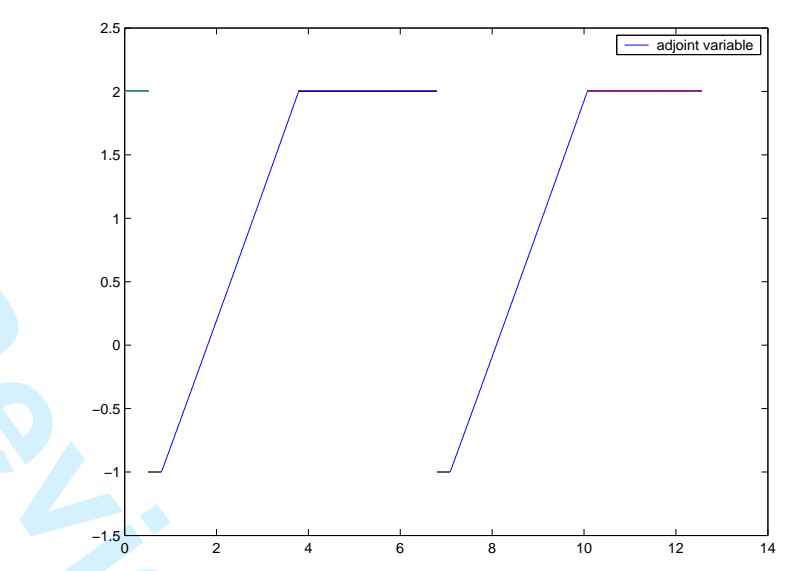

Figure 6. Optimal adjoint variable

In a second variant of Example 2 we show a similar case where used products are purchased. Therefore, let $b=10$. Synchronization is not optimal in the interval $[2.099,4.164]$, and additional manufacturing under zero inventories is required for $[0,0.253]$ and $[2.889,2 \pi]$. The application of our algorithm to this instance reveals that in the interval $[0,0.253]$ returns are acquired and remanufactured at the maximum rate of 17.5 and excessive demands are manufactured, returns and demands are synchronized in [0.253, 0.524], demands are remanufactured and excessive returns disposed of in $[0.524,0.847]$, positive recoverables inventory is optimal for $[0.847,3.833]$ where in $[0.847,3.342]$ only autonomous returns are collected and additional returns are bought by acquisition activities in $[3.342,3.833]$. In $[3.833,2 \pi]$, again returns are acquired and remanufactured with a rate of 17.5 and excessive demands are manufactured. Thereafter, the same policy is repeated. The performance of the policies is given in Table 3.

\section{Summary and outlook}

In this paper we have presented a dynamic optimisation approach to coordinate manufacturing and remanufacturing using both, inventory and pricing mechanisms to control 
Table 3. Relevant costs heuristics

\begin{tabular}{lccccc}
\hline & Optimal & AUTO1 & AUTO2 & SYN & STA \\
\hline $\mathrm{b}=5$ & 144.50 & 159.81 & 144.55 & 693.56 & 159.81 \\
\hline $\mathrm{b}=10$ & 140.85 & 159.81 & 144.55 & 353.52 & 155.01 \\
\hline
\end{tabular}

the product return and recovery process. The general framework of optimal control allows to obtain some analytical relationships between the optimal recovery and buy back strategy. However, the overall optimal policy has to be determined numerically. Incentives for storage of returns are either the collection of excessive autonomous returns or a cost beneficial buy-back in expectation of less favorable buy-back conditions or increasing demands. Additionally, our analysis enables us to show under which conditions some simple heuristics are optimal. Using a passive return acquisition policy is in general not close to optimality. This heuristic leads only to good results if the number of products brought back autonomously is already quite large. Otherwise large improvements can be achieved through a proactive return acquisition management.

There are several extensions to generalize the results. Concerning the return response functions, more general functions rather than a linear relationship can be analyzed. Further, carry over effects of past decisions, especially the market size, can be incorporated by introducing a second stock variable (market size) which is influenced by sales and take back and then, the response function depends on the market size and the price. Another extension is to consider joint sales and return pricing where in addition, price bundling effects with a joint price for a sale in case an item is returned can be analysed. The presented approach can also be generalized with respect to the underlying market structure. As a first extension one can think of two separate markets for new and remanufactured products that will have different sales prices and to analyse the associated market segmentation effects. Further, the impact of having several players, e.g. original equipment manufacturers and competing remanufacturing firms that both aim to acquire used products is another stream for future research.

\section{References}

Bakal, I.S. and Akcali, E., 2006. Effects of Random Yield in Remanufacturing with Price-Sensitive Supply and Demand. Production and Operations Management, 15 (3), 407-420.

Dekker, R., Fleischmann, M., Inderfurth, K., and Van Wassenhove, L.N., 2004. Reverse Logistics - Quantitative Models for Closed-Loop Supply Chains. Berlin Heidelberg New York: Springer.

Dockner, E. and Jørgensen, S., 1988. Optimal advertising policies for diffusion models of new product innovations in monopolistic situations. Management Science, 34 (1), $119-130$.

Eliashberg, J. and Steinberg, R., 1993. Marketing-Production Joint Decision Making. In: Eliashberg, J. and G.L. Lilien, eds. Handbooks in Operations Research and Management Science. Vol. 5: Marketing. Amsterdam: North-Holland, 827-880.

Feichtinger, G., Hartl, R.F., and Sethi, S.P., 1994. Dynamic Optimal Control Models in Advertising: Recent Developments. Management Science, 40 (2), 195-226.

Fleischmann, M., Bloemhof-Ruwaard, J.M., Dekker, R., van der Laan, E.A., van Nunen, 


\section{Cctober 23,2010
Page $\mathbf{1 7}$ of $\mathbf{2 2}$ \\ International Journal of Production Research}

International Journal of Production Research

J.A.E.E., and van Wassenhove, L.N., 1997. Quantitative models for reverse logistics: A review. European Journal of Operational Research, 103 (1), 1-17.

Fleischmann, M., 2001. Quantitative Models for Reverse Logistics. Berlin Heidelberg New York: Springer.

Galbreth M.R. and Blackburn J.D., 2006. Optimal Acquisition and Sorting Policies for Remanufacturing. Production and Operations Management, 15 (3), 384-392.

Guide Jr., V.D.R., Jayaraman, V., 2000. Product acquisition management: current industry practice and a proposed framework. International Journal of Production Research, 38 (16), 3779-3800.

Guide, D. and Van Wassenhove, L.N., 2001. Managing Product Returns for Remanufacturing. Production and Operations Management, 10 (2), 142-155.

Guide Jr., V.D.R., Teunter, R.H., and Van Wassenhove, L.N., 2003. Matching demand and Supply to Maximize Profits from Remanufacturing. Manufacturing \& Service Operations Management, 5 (4), 303-316.

Kalish, S., 1983. Monopolist pricing with dynamic demand and production costs. Marketing Science, 2 (2), 135-159.

Kamien, M.I. and Schwartz, N.L., 1991. Dynamic optimization: the calculus of variations and optimal control in economics and management. 2nd. ed. Amsterdam: NorthHolland.

Lariviere, M.A., 1999. Supply Chain Contracting and Coordination with Stochastic Demand. In: Tayur, S., Ganeshan, R. and M. Magazine, eds., Quantitative Models for Supply Chain Management. Boston: Kluwer, 231-268.

Mesak, H.I., 1996. Modelling Monopolist Pricing and Protection Period Decisions for New Products under Warranty. Optimal Control Applications \& $\&$ Methods, 17, 231-252.

Mesak, H.I. and Clark, J.W., 1998. Monopolist Optimum Pricing and Advertising Policies for Diffusion Models of New Product Innovations. Optimal Control Applications $\&$ Methods, 19 (2), 111-136.

Minner, S., 2003. Multiple Supplier Inventory Models in Supply Chain Management: A Review. International Journal of Production Economics, 81-82, 265-279.

Minner, S. and Kleber, R., 2001. Optimal control of production and remanufacturing in a simple recovery model with linear cost functions. OR Spektrum, 23 (1), 3-24.

Noble, P.M. and Gruca, T.S., 1999. Industrial Pricing: Theory and Managerial Practice. Marketing Science, 18 (3), 435-454.

Rao, V.R., 1993. Pricing Models in Marketing. In: Eliashberg, J. and G.L. Lilien, eds. Handbooks in Operations Research and Management Science. Vol. 5: Marketing. Amsterdam: North-Holland, 517-552.

Sasikumar, P. and Kannan, G., 2008. Issues in reverse supply chains, Part I: End-of life product recovery and inventory management - An overview. International Journal of Sustainable Engineering, 1 (3), 154-172.

Simon, H., 1989. Price Management. Amsterdam: Elsevier.

Vadde, S., Kamarthi, S.V., and Gupta, S.M., 2007. Optimal pricing of reusable and recyclable components under alternative product acquisition mechanisms. International Journal of Production Research, 45 (18-19), 4621-4652.

\section{Appendix A. Proof of Theorem 2.1}

In order to solve the optimization problem (6) we have to minimize a non-linear (quadratic), convex function subject to linear constraints. Using a Lagrange-approach 
we define the Lagrange-function

$$
L\left(p, u, \mu_{1}, \mu_{2}\right)=\left(-c_{p}+c_{u}-c_{z}\right) u+\left(c_{z}+p\right)(a+b p)+\mu_{1}(u-d)+\mu_{2}(u-a-b p) .
$$

Due to the convexity of the objective function, the following Kuhn-Tucker conditions are necessary and sufficient for an optimal solution.

$$
\begin{aligned}
\frac{\partial L}{\partial p}=\left(a+2 b p+b c_{z}\right)-b \mu_{2} & \geq 0 \\
p \cdot \frac{\partial L}{\partial p}\left(p, u, \mu_{1}, \mu_{2}\right) & =0 \\
\frac{\partial L}{\partial u}=-c_{p}-c_{z}+c_{u}+\mu_{1}+\mu_{2} & \geq 0 \\
u \cdot \frac{\partial L}{\partial u}\left(p, u, \mu_{1}, \mu_{2}\right) & =0 \\
\frac{\partial L}{\partial \mu_{1}}=u-d & \leq 0 \\
\mu_{1}(u-d) & =0 \\
\frac{\partial L}{\partial \mu_{2}}=u-a-b p & \leq 0 \\
\mu_{2}(u-a-b p) & =0 \\
p, u, \mu_{1}, \mu_{2} & \geq 0
\end{aligned}
$$

For the two decision variables $u, p$ we identify the following cases:

- $u=p=0$

$\mu_{1}=\mu_{2}=0$ from (A6) and (A8), $x=d, z=a$. Further, $c_{p}+c_{z}-c_{u} \leq 0,-c_{z} \leq a / b$ from (A3) and (A1). This identifies region E.

- $u=0, p>0$

$\mu_{1}=\mu_{2}=0$ from (A6) and (A8), $x=d, p=-\left(a+b c_{z}\right) /(2 b), z=\frac{a-b c_{z}}{2}$. Further, $c_{p}+c_{z}-c_{u} \leq 0,-c_{z}>a / b$ from (A3) and due to $p>0$. This identifies region $\mathrm{F}$.

- $0<u<\min \{d, a+b p\}, p=0$

$\mu_{1}=\mu_{2}=0$ from (A6) and (A8), $c_{p}+c_{z}-c_{u}=0,-c_{z} \leq a / b$ from (A3) and (A1). All $u, x$ combinations have the same objective function value. Since this is a boundary case, it can be regarded as a special case of region $\mathrm{E}$ with $u=0$.

- $0<u<\min \{d, a+b p\}, p>0$

$\mu_{1}=\mu_{2}=0$ from (A6) and (A8), $p=-\left(a+b c_{z}\right) /(2 b)$ from (A2). Further, $c_{p}+c_{z}-$ $c_{u}=0$ from (A4) and $-c_{z}>a / b$ from $p>0$. All $u, x$ combinations have the same objective function value. Since this is a boundary case, it can be regarded as a special case of region $\mathrm{F}$ with $u=0$.

- $u=d, u<a+b p, p=0$

$\mu_{2}=0$ from (A7) and (A8), $\mu_{1}=c_{p}+c_{z}-c_{u}$ from (A4) and $-c_{z} \leq a / b$ from (A1). This defines region $\mathrm{G}$ in the case of $a>d$ (see Figure 2).

- $u=d, u=a+b p, p=0$

$\mu_{2} \leq\left(a+b c_{z}\right) / b$ from (A1), i.e. $-c_{z} \leq a / b, \mu_{1}+\mu_{2} \geq c_{p}+c_{z}-c_{u}$ from (A3). Further, $x=0$ and $z=0$. This is a special case of region $\mathrm{G}$ if $a=d$ holds.

- $0<u<d, u=a+b p, p=0$ 


\section{Appendix B. Proof of Theorem 3.1}

Due to Pontryagin's Maximum Principle (for details see Kamien and Schwartz 1991) the solution of (8) is equivalent to the maximum of the corresponding current value static Hamilton-function subject to the non-negativity constraints for each point in time where the Hamilton-function is defined as

$$
\begin{aligned}
H(p(t), u(t), z(t), \lambda(t)):= & \left(c_{p}-c_{u}\right) u(t)-c_{z} z(t)-p(t) \cdot(a(t)+b(t) p(t))-h y(t) \\
& +\lambda(t) \cdot(a(t)+b(t) p(t)-u(t)-z(t))
\end{aligned}
$$

and $\lambda(t)$ is the adjoint variable to the movement of the inventory state (7). Maximizing (B1) subject to the given non-negativity constraints can be done using a Lagrange approach with the following Lagrange-function

$$
\begin{array}{r}
L\left(p(t), u(t), z(t), \lambda(t), \mu_{1}(t), \mu_{2}(t), \mu_{3}(t), \mu_{4}(t), \mu_{5}(t)\right):=H(p(t), u(t), z(t), \lambda(t) \\
+\mu_{1}(t) y(t)+\mu_{2}(t) p(t)+\mu_{3}(t) u(t)+\mu_{4}(t) z(t)+\mu_{5}(t)(d(t)-u(t))
\end{array}
$$

where $\mu_{1}(t), \mu_{2}(t), \mu_{3}(t), \mu_{4}(t)$, and $\mu_{5}(t)$ are the Lagrange multipliers associated with the non-negativity of inventory, price, remanufacturing, disposal, and manufacturing. We obtain the following necessary conditions, results, and equations:

(1) Maximization of the Hamilton-function (B1) reveals the optimal decisions:

- optimal remanufacturing decision

$$
u^{*}(t)= \begin{cases}d(t) & \lambda(t)<c_{p}-c_{u} \\ \text { undet. } & \lambda(t)=c_{p}-c_{u} \\ 0 & \lambda(t)>c_{p}-c_{u}\end{cases}
$$


- optimal disposal decision

$$
z^{*}(t)= \begin{cases}\infty & \lambda(t)<-c_{z} \\ \text { undet. } & \lambda(t)=-c_{z} \\ 0 & \lambda(t)>-c_{z}\end{cases}
$$

- optimal price

$$
p^{*}(t)=\arg \max \left\{H\left(p(t), u^{*}(t), z^{*}(t)\right)\right\}
$$

(2) Maximization of the Lagrange-function (B2) leads to the following equations:

$$
\begin{gathered}
\frac{\partial L}{\partial p}=0 \Leftrightarrow(\lambda(t)-p(t)) b(t)-(a(t)+b(t) p(t))+\mu_{2}(t)=0 \\
\frac{\partial L}{\partial u}=0 \Leftrightarrow c_{p}-c_{u}-\lambda(t)+\mu_{3}(t)-\mu_{5}(t)=0 \\
\frac{\partial L}{\partial z}=0 \Leftrightarrow-c_{z}-\lambda(t)+\mu_{4}(t)=0
\end{gathered}
$$

(3) The adjoint variable has to satisfy the differential equation:

$$
\dot{\lambda}(t)=\rho \lambda(t)+h-\mu_{1}(t)
$$

(4) Complementary Slackness conditions have to hold:

$$
\begin{aligned}
& \mu_{1}(t) y(t)=0, \mu_{1}(t) \geq 0, \quad \mu_{2}(t) p(t)=0, \mu_{2}(t) \geq 0, \quad \mu_{3}(t) u(t)=0, \mu_{3}(t) \geq 0 \\
& \mu_{4}(t) z(t)=0, \mu_{4}(t) \geq 0 \quad \mu_{5}(t)(d(t)-u(t))=0, \mu_{5}(t) \geq 0
\end{aligned}
$$

(5) The adjoint variable $\lambda(t)$ is a piecewise continuous function which can have jumps at certain points in time. Possible candidates are time points $\theta$ with $a(\theta)=d(\theta)$ and $y(\theta)=0$.

Since the objective function is separable in the decision variables, linear in $u$ and $z$, and quadratic and convex in $p$, the necessary conditions are sufficient. Using (B6) the optimal buy back price can be expressed as a function of the adjoint variable $\lambda(t)$ which is the shadow price (value of an additional return) at time $t$.

$$
p^{*}(t)=\left\{\begin{array}{lll}
\frac{\lambda(t)}{2}-\frac{a(t)}{2 b(t)} & \text { if } & \lambda(t)>\frac{a(t)}{b(t)} \\
0 & \text { if } & \lambda(t) \leq \frac{a(t)}{b(t)}
\end{array}\right.
$$

The resulting optimal return quantity becomes

$$
r^{*}(t)=\left\{\begin{array}{lll}
\frac{a(t)}{2}+\frac{\lambda(t) b(t)}{2} & \text { if } & \lambda(t)>\frac{a(t)}{b(t)} \\
a(t) & \text { if } & \lambda(t) \leq \frac{a(t)}{b(t)}
\end{array} .\right.
$$


If $y(t)=0$ for a time-interval with positive length, then $\dot{y}(t)=0$ and $r^{*}(t)=u^{*}(t)+$ $z^{*}(t)$ from (7) which implies $0 \leq u^{*}(t) \leq r^{*}(t)$ and $0 \leq z^{*}(t) \leq r^{*}(t)$. We have to distinguish the following 4 subcases.

a) $u^{*}(t)<\min \left\{d(t), r^{*}(t)\right\}, z^{*}(t)>0$

Due to (B10) and (B8) we get $\lambda(t)=-c_{z}$. On the other hand it follows from (B3) that $\lambda(t) \geq c_{p}-c_{u}$. This contradicts assumption $(\alpha 1)$ and therefore this cannot be optimal.

b) $u^{*}(t)=r^{*}(t)<d(t), z^{*}(t)=0$.

Then, $\lambda(t)=c_{p}-c_{u}$ from (B3) and the optimal return rate from (B12) is

$$
r^{*}(t)=\left\{\begin{array}{ll}
\frac{a(t)}{2}+\frac{\left(c_{p}-c_{u}\right) b(t)}{2} & \text { if } \quad c_{p}-c_{u}>\frac{a(t)}{b(t)} \\
a(t) & \text { if } \quad c_{p}-c_{u} \leq \frac{a(t)}{b(t)}
\end{array} .\right.
$$

Since $r^{*}(t)<d(t)$ this is only possible if $d(t)>\frac{a(t)}{2}+\frac{b(t)}{2}\left(c_{p}-c_{u}\right)$. Since backorders are not allowed we additionally have $x^{*}(t)=d(t)-r^{*}(t)$.

c) $u^{*}(t)=r^{*}(t)=d(t), z^{*}(t)=0$.

Then, $\lambda(t)<c_{p}-c_{u}$ from (B3) and to ensure $r^{*}(t)=d(t)$ we need

$$
\lambda(t)=\frac{2 d(t)-a(t)}{b(t)} .
$$

Due to $z^{*}(t)=0$ we get $\lambda(t)>-c_{z}$. Therefore, this case is only possible if $a(t)<d(t)$ and $d(t) \geq \frac{a(t)}{2}-c_{z} \frac{b(t)}{2}$ and $d(t) \leq \frac{a(t)}{2}+\frac{b(t)}{2}\left(c_{p}-c_{u}\right)$. It is further optimal not to manufacture $\left(x^{*}(t)=0\right)$.

d) $u^{*}(t)=d(t), z^{*}(t)=r^{*}(t)-d(t),\left(r^{*}(t)>d(t)\right)$,

As a consequence of (B4), $\lambda(t)=-c_{z}$ and the returns are given by

$$
r^{*}(t)=\left\{\begin{array}{ll}
\frac{a(t)}{2}-c_{z} \frac{b(t)}{2} & \text { if }-c_{z}>\frac{a(t)}{b(t)} \\
a(t) & \text { if }-c_{z} \leq \frac{a(t)}{b(t)}
\end{array} .\right.
$$

Due to assumption $(\alpha 2)$ we have to exclude $-c_{z}>\frac{a(t)}{b(t)}$ and to ensure $r^{*}(t)>d(t)$ we require $a(t)>d(t)$.

\section{Appendix C. Proof of Theorem 3.2}

In case of $y(t)>0$ we get $\mu_{1}(t)=0$ from (B10) and the solution of the differential equation (B9) can be obtained as

$$
\lambda(t)=-\frac{h}{\rho}+\left(\lambda\left(t_{0}\right)+\frac{h}{\rho}\right) e^{\rho\left(t-t_{0}\right)}
$$

where $t_{0}$ denotes the starting or ending point of an interval with $y(t)>0$. Due to our assumption $h>\rho c_{z}$ and using $\lambda(t) \geq-c_{z}$ we find

$$
\dot{\lambda}(t)=\left(\rho \lambda\left(t_{0}\right)+h\right) e^{\rho\left(t-t_{0}\right)}>0 .
$$


Assume $u(t)<d(t)$. This requires $\lambda(t) \geq c_{p}-c_{u}$ which is not possible for an interval with positive length because $\dot{\lambda}(t)>0$ and $\lambda(t)>c_{p}-c_{u}$ requires $u(t)=0$ which would imply that recoverables inventory will never be depleted to zero which is required as the final inventory level.

Assume $z(t)>0$. Then, $\mu_{4}(t)=0, \lambda(t)=-c_{z}$ from (B4), and $\dot{\lambda}(t)=0$ which contradicts $(\mathrm{C} 2)$. Summarizing, in an interval with $y(t)>0$, we have $u^{*}(t)=d(t)$ and $z^{*}(t)=x^{*}(t)=0$.

From $\lambda(t)<c_{p}-c_{u}$ it follows that there exists an upper limit for the optimal return rate,

$$
r^{*}(t)<\frac{a(t)+b(t)\left(c_{p}-c_{u}\right)}{2}
$$

The lower limit is $a(t)$. Depending on the value of $\lambda(t)$, i.e. whether $\lambda(t) \leq a(t) / b(t)$ or $\lambda(t)>a(t) / b(t)$, the buy back price is either zero or positive.

\section{Appendix D. Proof of Proposition 3.4}

Under the assumption of $y(t)=0$ for all $t \in\left[\theta-\epsilon_{1}, \theta+\epsilon_{2}\right]$ and $-c_{z}<\frac{2 d(\theta)-a(\theta)}{b(\theta)}$ we get $\lambda(t)=\frac{2 d(t)-a(t)}{b(t)}>-c_{z}$ for all $t \in\left[\theta, \theta+\epsilon_{2}\right]$ and $\lambda(t)=-c_{z}$ for all $t \in\left[\theta-\epsilon_{1}, \theta[\right.$. This is equivalent with an upward jump of the adjoint variable, which is not allowed due to the optimality conditions. Therefore, collection of returns has to start before $\theta$ which means $y(t)>0$ for all $t \in\left[\theta-\epsilon_{1}, \theta+\epsilon_{2}\right]$. Otherwise, if $\frac{2 d(t)-a(t)}{b(t)} \leq-c_{z}$ for all $t \in\left[\theta, \theta+\epsilon_{2}\right]$ there is no need to build up inventories.

\section{Appendix E. Proof of Proposition 3.5}

Under the assumption of

$$
\frac{a(t)}{2}-c_{z} \frac{b(t)}{2} \leq d(t)<\frac{a(t)}{2}+\frac{b(t)}{2}\left(c_{p}-c_{u}\right) .
$$

and $y(t)=0$ we have $\lambda(t)=\frac{2 d(t)-a(t)}{b(t)}$ and $\dot{\lambda}(t)=\frac{b(t)(2 \dot{d}(t)-\dot{a}(t))-2 d(t) \dot{b}(t)-a(t) \dot{b}(t)}{b^{2}(t)}$. Since $\mu_{1}(t) \geq 0$ we know from (B9) that $\dot{\lambda}(t) \leq \rho \lambda(t)+h$. Therefore, it is clear that $y(t)=0$ cannot appear in an optimal solution if

$$
\dot{\lambda}(t)>\rho \lambda(t)+h
$$

such that for all time points satisfying (13) a positive inventory is required. 